\title{
Lymphbahnen am Bein
}

\section{Lymph channels in the leg}

Autor

Erich Brenner (D)

Institut

Institut für Klinisch-Funktionelle Anatomie, Medizinische

Universität Innsbruck, Österreich

Schlüsselwörter

Lymphologie, Phlebologie

Key words

lymphology, phlebology

online publiziert 03.11 .2020

Bibliografie

Phlebologie 2020; 49: 340-350

DOI 10.1055/a-1246-5748

ISSN 0939-978X

(c) 2020. Thieme. All rights reserved.

Georg Thieme Verlag KG, Rüdigerstraße 14,

70469 Stuttgart, Germany

Korrespondenzadresse

Ao. Univ.-Prof. Dr. Erich Brenner, MME (Bern)

Institut für Klinisch-Funktionelle Anatomie

Medizinische Universität Innsbruck, Müllerstraße 59,

6020 Innsbruck, Österreich

erich.brenner@i-med.ac.at

\section{ZUSAMMENFASSUNG}

Zu den Komplikationen der Varizentherapie zählt auch das Lymphödem. Pittaluga und Chastanet fanden nach chirurgischer Intervention in nur 0,25\% ein Lymphödem. Flessenkämper und Mitarbeiter konnten zeigen, dass dieses, je nach verwendeter Therapiemethode, in 2,8\% (hohe Ligatur und Stripping) bis 9,2\% (endovenöse Laserablation) auftritt. Einer der möglichen Gründe für diese Lymphödeme könnte eine direkte Schädigung der (sub)inguinalen Lymphgefäße sein. In einer anatomischen Studie fand sich an einem Präparat eine feine „VSM-Entnahmenarbe“ am medialen linken Oberschenkel, vermutlich nach einer Crossektomie. Alle Kollektoren, die die Narbe durchquerten, obliterierten an der Narbe, während sie auf die großen inferolateralen Lymphknoten zogen. Seitlich der Narbe hatten sie ein normales Aussehen und konnten rekanüliert werden. Bislang hat die (anatomische) Forschung diesem Aspekt wenig Beachtung geschenkt; dabei zeigte sich jedoch, dass auch unterhalb der Fascia saphena, parallel zur V. saphena magna, mehrere Lymphgefäße verlaufen.
In der hier präsentierten explorativen anatomischen Untersuchung wurden 20 untere Extremitäten von 10 Individuen verwendet.

An den rechten Präparaten wurde eine akribische und soweit wie möglich schichtweise Präparation der Subinguinalregion durchgeführt. Die linken Präparate wurden gefroren und zur Hälfte longitudinal und zur anderen Hälfte transversal geschnitten. Die so erhaltenen Schnitte wurden auf den genauen Verlauf der Fascia lata, der Fascia cribrosa und der Fascia saphena untersucht und die genaue Lage der Lymphknoten und -bahnen wurde festgestellt.

Eine extensive Intervention an der proximalen V. saphena magna, sei es bei der klassischen Crossektomie oder bei diversen endovenösen Verfahren, deren jeweiliges Agens über die Venenwand selbst hinausreichen kann, kann zu einer zumindest teilweisen Schädigung von begleitenden Lymphgefäßen führen. Zumeist wird wohl die Gesamttransportkapazität der oberflächlichen Lymphbahnen des Beins nicht unter die anfallende Lymphlast gesenkt; jedoch kann eine vorbestehende Beeinträchtigung oder auch eine später auftretende weitere Minderung der Gesamttransportkapazität in einem Lymphödem resultieren.

\section{ABSTRACT}

Lymphedema is one of the complications of varicose vein therapy. Pittaluga and Chastanet found lymphedema in only $0.25 \%$ after surgical intervention. Flessenkämper and colleagues were able to show that this occurs in $2.8 \%$ (high ligature and stripping) to $9.2 \%$ (endovenous laser ablation), depending on the therapy method used. One of the possible reasons for this lymphedema could be a direct damage of the (sub)inguinal lymphatic vessels. In an anatomical study, a fine "VSM removal scar" was found on the medial left thigh, probably after a crossectomy. All collectors that passed through the scar obliterated at the scar while moving to the large inferolateral lymph nodes. Laterally to the scar they had a normal appearance and could be recannulated. Up to now, (anatomical) research has paid little attention to this aspect; however, it has been shown that several lymph vessels run underneath the saphenous fascia, parallel to the great saphenous vein.

In the exploratory anatomical study presented here, twenty lower extremities of ten individuals were used.

The right-hand specimens were meticulously dissected in layers of the subinguinal region as far as possible. The left specimens were frozen and cut half longitudinally and half trans- 
versely. The resulting sections were examined for the exact course of the fascia lata, the cribrous fascia and the saphenous fascia and the exact location of the lymph nodes and pathways was determined.

Extensive intervention on the proximal saphenous vein, whether in classical crossectomy or in various endovenous procedures, the respective agent of which may extend be- yond the venous wall itself, can lead to at least partial damage to accompanying lymph vessels. In most cases, the total transport capacity of the superficial lymph channels of the leg is not reduced below the lymphatic load; however, a pre-existing impairment or a later occurring further reduction of the total transport capacity can result in lymphedema.

\section{Einleitung}

Zu den Komplikationen der Varizentherapie zählt auch das Lymphödem. Pittaluga und Chastanet fanden nach chirurgischer Intervention in nur 0,25\% ein Lymphödem [1]. Flessenkämper und Mitarbeiter konnten zeigen, dass dieses, je nach verwendeter Therapiemethode, bei der ersten Kontrolle nach OP bei 4 Patienten der Krossektomie- und Strippinggruppe sowie in der Krossektomie- und endoluminalen LASER-Ablationsgruppe (EVLA) und bei 2 Patienten der EVLA-Gruppe (ohne chirurgische Krossektomie) auftrat. Bei der Kontrolle nach 2 Monaten fanden sich Ödeme in 2,8\% (hohe Ligatur und Stripping) bis 9,2\% (EVLA). Alle diese Werte waren nicht signifikant unterschiedlich zwischen den Gruppen; längerfristige Ergebnisse sind nicht publiziert [2] (siehe auch > Tab. 1). Eine der möglichen Gründe für diese Lymphödeme könnte eine direkte Schädigung der (sub)inguinalen Lymphgefäße sein. In einer anatomischen Studie fand sich an einem Präparat eine feine „VSM-Entnahme-Narbe“ am medialen linken Oberschenkel [3]. Alle Kollektoren obliterierten an der Narbe, während sie in Richtung auf die großen inferolateralen Lymphknoten zogen. Seitlich der Narbe hatten sie ein normales Aussehen und konnten rekanüliert werden. Bislang hat die (anatomische) Forschung diesem Aspekt wenig Beachtung geschenkt [4-6]; dabei zeigte sich jedoch, dass auch unterhalb der Fascia saphena, parallel zur V. saphena magna, mehrere Lymphgefäße verlaufen [5].

\section{Faszien am Bein}

Einleitend müssen die grundlegenden Faszienverhältnisse dargestellt und für Bauch und Bein vereinheitlicht werden ( $\triangleright$ Tab. 2).

Der gesamte Körper wird - bis auf wenige Ausnahmen - von einer oberflächlichen Bindegewebshülle, der Oberflächenfaszie, Fascia investiens (von Lat. investīre: bekleiden), umhüllt. Diese Fascia investiens ist mit den Septa intermuscularia mit den darunterliegenden Skelettelementen verbunden und bildet mit diesen osteofibröse Räume, die als Kompartimente bezeichnet werden.

Die Fascia investiens stellt zudem auch die innerste Basis der Subcutis dar. Von der Fascia investiens steigen mehr oder minder vollständige Septen zur Cutis auf, um einerseits diese mit der Fascia investiens zu verbinden und andererseits die subkutanen Fettläppchen zu gliedern. Die Subkutis-Septen selbst sind wiederum durch oberflächenparallel verlaufende Bindegewebslamellen untereinander verbunden, die Fascia subcutanea. Diese Fascia subcutanea stellt an den meisten Stellen eine dreidimensionale, siebartige Struktur dar; daher auch an manchen Stellen die Bezeichnung Fascia cribrosa oder Lamina cribrosa.
Die großen Körperhöhlen werden zudem von jeweils einer eigenen Hüllfaszie ausgekleidet; im Bauchraum ist dies die Fascia transversalis und im Thorax die Fascia thoracica interna.

Neben diesen Oberflächen- und Hüllfaszien besitzen jedes Organ und jeder Muskel eine eigene Organ- bzw. Muskelfaszie. Liegt eine Muskelfaszie einer Oberflächenfaszie von innen her an, so verschmelzen diese beiden Bindegewebsblätter miteinander, sodass es den Anschein hat, dass dieser Muskel nach außen nur von der Fascia investiens bedeckt sei.

Das große Problem der Faszien, nicht nur am Bauch und am Bein, ist die sehr uneinheitliche Terminologie, was sich leider auch in der „offiziellen“ Terminologia Anatomica [7] niederschlägt.

Die eigentliche Oberflächenfaszie des Bauchs ist die Fascia investiens (profunda) abdominis [7], üblicherweise als Fascia abdominalis superficialis, lamina profunda, bezeichnet; sie bedeckt den M. obliquus externus abdominis und die Rektusscheide, mit der sie unverschieblich verwachsen ist. Am Anulus inguinalis superficialis geht sie als dünne Fascia spermatica externa auf das Lig. teres uteri ( + ) bzw. den Funiculus spermaticus ( $\left.\sigma^{\top}\right)$ über. Sie wird nach Antonio Scarpa (1752-1832), einem italienischen Anatomen und Chirurgen, auch als ScARPA-Faszie tituliert. Die Fascia investiens abdominis setzt sich auf dem Oberschenkel als Fascia lata fort.

Bei der Fascia lata fallen offizielle Terminologie und übliche lateinische Bezeichnung zusammen; korrekterweise müsste sie als Fascia investiens femoris bezeichnet werden. Auch auf sie findet gelegentlich der eponymische Begriff „SCARPA-Faszie“ Anwendung. In der Fascia lata bildet der Hiatus saphenus die Durchtrittstelle für die V. saphena magna. Am Unterschenkel wird die Fascia investiens cruris dann als Fascia cruris bezeichnet; auch bei ihr fallen übliche lateinische Bezeichnung und offizielle anatomische Terminologie zusammen.

Im subkutanen Fettgewebe des Bauchs (Panniculus adiposus abdominis) findet sich eine wabenartig perforierte Bindegewebsstruktur, die den offiziellen Namen Fascia investiens superficialis (abdominis) trägt [7], üblicherweise aber als Fascia abdominalis superficialis, lamina superficialis, bezeichnet wird. Eine frühere durchaus sinnvolle - Bezeichnung war auch „Fascia subcutanea (abdominis)“. Nach dem niederländischen Anatomen und Chirurgen Peter Camper (1722-1789) wird sie als CAMPER-Faszie tituliert.

Diese Fascia investiens superficialis (abdominis) setzt sich natürlich auch auf dem Oberschenkel fort und bildet dort eine Fascia subcutanea femoris, besitzt aber keine offizielle Bezeichnung; einzig im Bereich über dem Hiatus saphenus wird sie als Fascia cribrosa [7] bezeichnet. 
- Tab. 1 Lymphödem als Komplikation der Varizentherapie.

\begin{tabular}{|c|c|c|}
\hline $\begin{array}{l}\text { Komplika- } \\
\text { tionsrate }\end{array}$ & Therapieform & Referenz \\
\hline $0,6 \%$ & $\begin{array}{l}\text { Sklerotherapie } \\
\text { (PSTS) }\end{array}$ & Cavezzi, Frullini, Ricci et al. [27] \\
\hline $0,9 \%$ & $\begin{array}{l}\text { Crossektomie } \\
\text { (mit Barriere) }\end{array}$ & $\begin{array}{l}\text { De Maeseneer, Philipsen, } \\
\text { Vandenbroeck et al. [28] }\end{array}$ \\
\hline $1,3 \%$ & $\begin{array}{l}\text { Crossektomie \& } \\
\text { Stripping }\end{array}$ & $\begin{array}{l}\text { De Maeseneer, Vandenbroeck, } \\
\text { Lauwers et al. [29] }\end{array}$ \\
\hline $6,9 \%$ & $\begin{array}{l}\text { Crossektomie \& } \\
\text { Stripping }\end{array}$ & \multirow[t]{2}{*}{$\begin{array}{l}\text { Figueiredo, Araujo, Barros } \\
\text { et al. [30] }\end{array}$} \\
\hline $0,0 \%$ & $\begin{array}{l}\text { Sklerotherapie } \\
\text { (Polidocanol) }\end{array}$ & \\
\hline $2,8 \%$ & $\begin{array}{l}\text { Crossektomie \& } \\
\text { Stripping }\end{array}$ & \multirow[t]{3}{*}{$\begin{array}{l}\text { Flessenkamper, Hartmann, } \\
\text { Stenger et al. [2] }\end{array}$} \\
\hline $9,2 \%$ & EVLA $(980 \mathrm{~nm})$ & \\
\hline $6,3 \%$ & $\begin{array}{l}\text { EVLA }(980 \mathrm{~nm}) \& \\
\text { Crossektomie }\end{array}$ & \\
\hline $0,2 \%$ & $\begin{array}{l}\text { Crossektomie \& } \\
\text { Stripping }\end{array}$ & Pittaluga and Chastanet [1] \\
\hline
\end{tabular}

- Tab. 2 Faszien an Bauch und Bein.

\begin{tabular}{|c|c|c|}
\hline $\begin{array}{l}\text { Übliche Bezeich- } \\
\text { nung(en) }\end{array}$ & $\begin{array}{l}\text { Terminologia } \\
\text { Anatomica [7] }\end{array}$ & Eponym \\
\hline $\begin{array}{l}\text { Fascia abdominalis } \\
\text { superficialis, lamina } \\
\text { superficialis; } \\
\text { Fascia subcutanea }\end{array}$ & $\begin{array}{l}\text { Fascia investiens } \\
\text { superficialis abdominis }\end{array}$ & Camper-Faszie \\
\hline $\begin{array}{l}\text { Fascia abdominalis } \\
\text { superficialis, lamina } \\
\text { profunda }\end{array}$ & $\begin{array}{l}\text { Fascia investiens } \\
\text { profunda abdominis }\end{array}$ & Scarpa-Faszie \\
\hline $\begin{array}{l}\text { Lamina cribrosa } \\
\text { fasciae latae; } \\
\text { Fascia subcutanea }\end{array}$ & Fascia cribrosa & $\approx$ Camper-Faszie \\
\hline \multicolumn{3}{|l|}{ Fascia saphena } \\
\hline Fascia lata & $\begin{array}{l}\text { Fascia lata } \\
(\approx \text { Fascia investiens femoris) }\end{array}$ & $\approx$ Scarpa-Faszie \\
\hline Fascia cruris & $\begin{array}{l}\text { Fascia cruris } \\
(\approx \text { Fascia investiens cruris })\end{array}$ & \\
\hline
\end{tabular}

Alberto Caggiati und Stefano Ricci haben 1997 erstmals das Kompartiment der V. saphena magna und damit die Fascia saphena am anatomischen Präparat beschrieben [8]. Sie schrieben, dass die V. saphena magna über den größten Teil ihrer Länge in einem engen Kompartiment verläuft, das tief durch die Muskelfaszie [Anm. des Autors: gemeint ist die Oberflächenfaszie, Fascia investiens] und oberflächlich durch eine Bindegewebslamelle, die vom Leistenband im anteromedialen Teil des Oberschenkels und an der medialen Seite des Unterschenkels herabsteigt, begrenzt wird. Diese beiden Faszien verschmelzen an den Grenzen des Kompartiments. Die
Adventitia der V. saphena magna ist durch dicke Bindegewebsstränge an beiden Faszien verankert. Bei Abb. 3A beschrieben sie, dass das Saphena-Kompartiment schmal sei, mit Ausnahme der Leistenregion, wo die Fascia saphena allmählich oberflächlich werde. Zusammenfassend gilt also: Distal des Hiatus saphenus wird die V. saphena magna von einer eigenen Fascia saphena bedeckt, wodurch sie vom eigentlichen Unterhautfettgewebe abgegrenzt wird und in einem eigenen Saphena-Kompartiment verläuft [9].

\section{Historische Beschreibungen und Darstellungen}

Die Beschreibungen und Darstellungen der oberflächlichen Lymphgefäße am Bein reichen historisch weit zurück. Eine erste zusammenfassende Beschreibung der Lymphknoten und der Lymphgefäße des Beins präsentierte 1786 William Cruikshank [10], der allerdings angibt, dass die Erstbeschreibung dieser Lymphgefäße auf Johannes van Horne zurückgeht. Cruikshank beschreibt schon damals, dass die oberflächlichen Lymphgefäße des Beins die beiden Venen begleiten, und nennt sie daher „vasa lymphatica venam saphenam majorem comitantia“ und „vasa lymphatica venam saphenam minorem comitantia“.

Cruishank beschreibt diese Lymphgefäße folgendermaßen ${ }^{1}$ (siehe auch - Abb. 1): "Sie bilden vier große Abteilungen; die erste entsteht zwischen der Großzehe und der zweiten Zehe, wo die V. saphena magna beginnt, und besteht aus sechs oder sieben Gefäßen; sie verlaufen über die Fußspitze mit dieser Vene in Richtung des vorderen Teils des Innenknöchels; von dort aus verlaufen sie, zusammen mit der Vene, in Richtung Knieinnenseite, wo sich weitere, noch zu beschreibende Gefäße anschließen. Die zweite Abteilung, die ich gesehen habe und die manchmal aus acht oder zehn Gefäßen besteht, entspringt etwa in der Mitte des inneren Fußrandes, verläuft hinter dem inneren Knöchel und schließt sich, über die Innenseite der Wade des Beins verlaufend, an die zuletzt beschriebenen Gefäße an der Innenseite des Knies an. Die dritte Abteilung, die aus fünf oder sechs Gefäßen besteht, entspringt in der Nähe der Kleinzehe, verläuft über die Außen- und Oberseite des Fußes in Richtung des äußeren Knöchels. Wenn sie sich diesem nähern, teilen sie sich in zwei Teile; ein Teil überquert den vorderen Teil des Schienbeins und geht ebenfalls zur Knieinnenseite, wo sie sich mit den beiden früheren Abteilungen verbinden. [...] Aus der Vereinigung dieser drei Abteilungen entsteht ein großer Plexus, bestehend aus vierzehn, sechzehn oder zwanzig Stämmen von Kollektoren, die noch immer die V. saphena magna begleiten, d. h. schräg von der Knieinnenseite zur Mitte der Leiste verlaufen; dort gehen sie gewöhnlich in die verschiedenen bereits beschriebenen Leistenlymphknoten über. [...] Es kommt häufig vor, dass zwei oder drei dieser Stämme an den Lymphknoten der Leistengegend vorbeigehen und erst dann in irgendeinen Lymphknoten einmünden, wenn sie unter dem Lig. inguinale durchgegangen sind. Ein Teil der letzten Abteilung, nämlich jene von der Kleinzehe kommende, schließt sich einer anderen an, die von der Mitte des äußeren Fußrandes herrührt, wo die V. saphena parva beginnt und die diese Vene begleitet, hinter dem äußeren Knöchel verläuft; von dort aus laufen sie an der Außenseite der Achillessehne, gehen danach zwischen den Köpfen des M. gastrocnemius und, in der Nähe der Stelle, wo diese

1 Übersetzungen durch den Autor; die Terminologie wurde der heutigen Terminologie angepasst. 


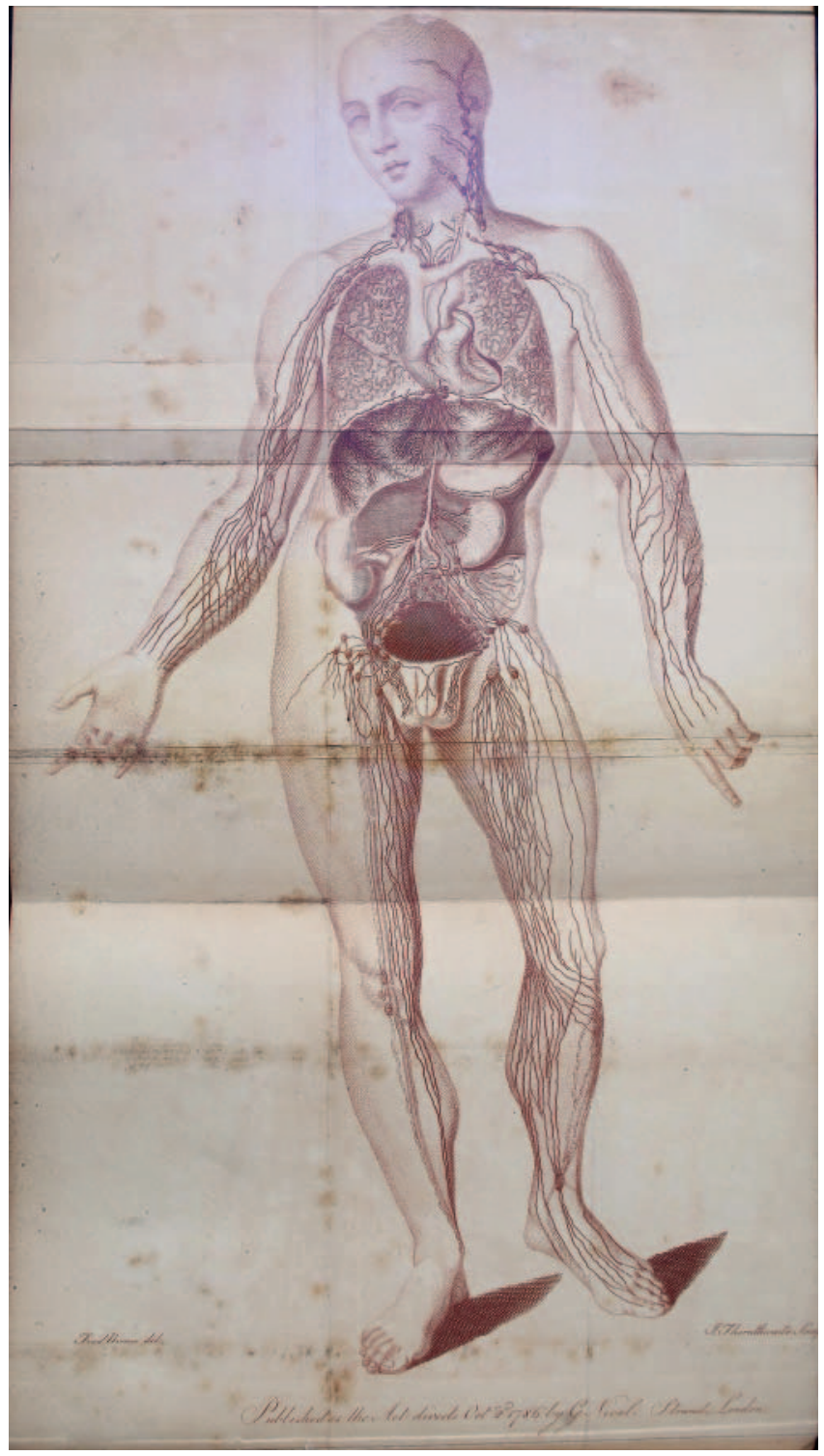

- Abb. 1 Bildtafel I aus Cruikshank (1786) [10].

[...] Das linke Bein stellt das kutane Saugadersystem in der erfolgreichsten Injektion dar, die ich [William Cruikshank] je mit diesen Gefäßen durchgeführt habe [...].

Das rechte Bein repräsentiert sowohl das kutane als auch das tiefsitzende Saugadersystem. [...] Die gestrichelten Linien stellen die tiefsitzenden Saugadern dar. [...]

an den Kondylen des Femurs entspringen, zwischen dessen Köpfen hinuntertauchend, enden in den bereits beschriebenen Lymphknoten der Kniekehle. [...] Diese sind nicht mehr als ein Fünftel so zahlreich wie die ersteren."

Die poplitealen Lymphknoten beschreibt Cruikshank so:

„Ich habe keine Lymphknoten in der unteren Extremität, unterhalb des Knies, gesehen. [...] Es sind selten mehr als drei, sie liegen dicht an der Kniekehlenarterie und sind, obwohl klein, keineswegs, wie Haller sagt, ,uti ultimae conglobatarum, ita minimae [etwa: „wie äußerst zusammengedrängt, so minimal klein"]'. Es gibt viele auf dem Mesokolon und an einer Vielzahl anderer Stellen, die viel kleiner sind als sie. Sie schwellen von Wunden an der Außenseite des Fußes, in der Fußsohle und von Wunden der Integumente an der Wade des Beins an. “

Zu den inguinalen Lymphknoten schreibt Cruikshank: „Die Lymphknoten der Leistengegend sind von einer ungewissen Anzahl, von acht, zehn oder zwölf bis zu zwanzig oder mehr. Haller beschreibt nur vier. Sie befinden sich hauptsächlich über der Faszie des Oberschenkels, obwohl mehrere von ihnen auch unter der Faszie liegen. Die letzteren liegen auf dem M. iliacus internus zwischen Quadriceps und Sartorius. Manchmal werden mehrere dieser Lymphknoten zu einem großen Lymphknoten zusammengefasst, der auf der Oberseite der A. femoralis liegt. Diejenigen, die der Symphysis pubica am nächsten liegen, gehören bei beiden Geschlechtern zu den Lymphgefäßen der Genitalien und werden bei Geschlechtskrankheiten zum Sitz von Bubonen. [...] Diejenigen, die sich an der Außenseite der letztgenannten Lymphknoten befinden, sind eher dazu geeignet, sich zu entzünden und zu vergrößern, und zwar aufgrund von [...] Wunden an der Innen-oder Oberseite des Fußes, von Wunden an der Innenseite des Knies oder von Wunden, die den Verlauf der V. saphena magna betreffen. Sie können durch Wunden an der Innenseite oder an einem wunden Teil des Beins und des Oberschenkels anschwellen. Ich kenne sie von Wunden am Gesäß und sogar von den entzündeten und blutenden Haufen am Rande des Afters. Wenn Pflaster auf die Haut in der Nähe der Wirbelsäule des Darmbeins aufgetragen wurden, die Geschwüre an den Integumenten verursachen, habe ich diese Lymphknoten auch wiederholt anschwellen sehen. Manchmal schwellen sie aus Sympathie mit einem entzündeten Hoden an und eitern sogar, obwohl es keine Verbindung zwischen ihnen durch Lymphgefäße gibt. Haller und Nuck lassen diese Drüsen manchmal bis zur Mitte des M. sartorius reichen. Ich habe selten Drüsen zwischen der Kniekehle und der Leiste gesehen, weder bei den kutanen noch bei den tiefen Lymphgefäßen.“

Auf die exemplarischen Präparationen von Paolo Mascagni, einem jungen Anatomen in Siena, der seine Befunde bereits 1787 in einer ausführlichen Monografie veröffentlichte [11], gehen auch die ersten dreidimensionalen Wachsmodelle zum Lymphgefäßsystem von Felice Fontana im Florentiner Museo La Specola zurück [12]. Interessanterweise beschreibt Mascagni zwar recht ausführlich die tiefen Lymphgefäße der unteren Extremitäten und auch die inguinalen Lymphknoten, nicht aber die oberflächlichen Lymphgefäße der unteren Extremitäten. Die Anordnung dieser Lymphgefäße in seiner Bildtafel IV ( $\triangleright$ Abb. 2) scheint aus heutiger Sicht eher willkürlich gewählt zu sein. Interessant ist aber auch, dass diese Monografie von Mascagni zusammen mit dem zuvor zitierten Buch Cruikshanks von Christian-Friedrich Ludwig schon 1789 ins Deutsche übersetzt wurde [13, 14].

\section{ABB. 2: BILDTAFEL IV AUS MASCAGNI (1787) [11]}

\section{Figura 1}

A Dissezierte Haut nach beiden Seiten umgeschlagen

- a Lymphstämme, über die die Injektionen durchgeführt wurden.

- b-g Stämme, die von außen an die Hinterseite ziehen.

- h i I m Stämme, die von der Innenseite zur unteren Hüfte nach hinten migrieren.

- 2.-17. Stämme, die aus der Innenseite des Unterschenkels hervorgehen. 


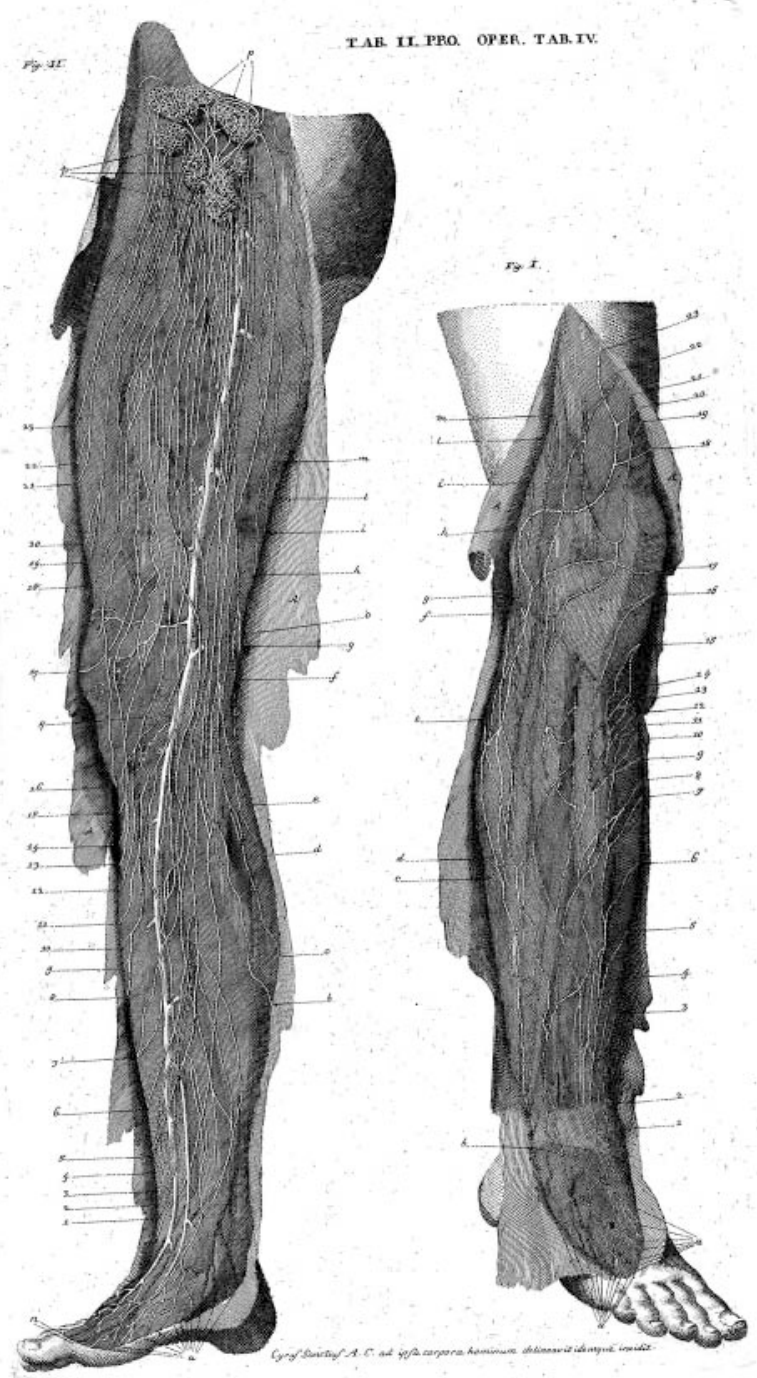

Abb. 2 Bildtafel IV aus Mascagni (1787) [11].

- 18.-23. Stämme, die aus der Innenseite zur unteren Hüfte nach vorne ziehen

\section{Figura 2}

A die gesamte Haut disseziert und nach außen umgeschlagen.

- a Neun Lymphstämme, über die die Quecksilberinjektion erfolgte, aus der Fußsohle hervorgehend.

- b-g Stämme, die von der Hinterseite des Unterschenkels kommend an die Innenseite ziehen.

- h il m Andere Stämme, die von der Hinterseite der Hüfte an die Innenseite migrieren.

- n Zwei Lymphstämme, die aus der Großzehe hervorgehen, über die Quecksilber injiziert wurde.

- 2.-17. Lymphstämme mit den gleichen Nummern wie in - Fig. 1 und die von der Vorder- an die Innenseite der Tibia ziehen.
- 18.-23. Stämme der gleichen Gefäße, die von der Außenan die Vorderseite der Hüfte ziehen.

- Stamm, der zwischen M. sarotius und M. vastus medialis zu den tiefen Lymphgefäßen zieht, um sich mit diesen zu verbinden.

- p Sechs Lymphknoten, in die die vorbeschriebenen Lymphgefäße einmünden.

- q V. saphena magna, die Seitenäste reseziert.

Den nächsten qualitativen Schritt erreichte Philibert Constant Sappey 1874 mit seinem umfassenden Werk zur Anatomie, Physiologie und Pathologie der Lymphgefäße [15]. Die Beschreibung der Lymphbahnen ist sehr ausführlich, und das Werk ist mit zahlreichen Kupferstichen illustriert ( $\triangleright$ Abb. $\mathbf{3})$.

\section{ABB. 3 BILDTAFEL V AUS SAPPEY (1874) [15] \\ Bildtafel $\mathrm{V}$}

Abb. 1 Lymphgefäße des anteromedialen Teils der unteren Extremität 1, Lymphatisches Netzwerk des Innenrandes des Fußes. Aus diesem Netzwerk wird eine beträchtliche Zahl von Stämmen hervorgehen, die sich unter der Haut schlängeln und sich zu Stämmen zusammenschließen.

2, Lymphatische Stämme aus dem peripheren Netz der Zehen und der vorderen Hälfte der Fußsohle.

3, Großer lymphatischer Stamm, der vom zentralen Teil der Plantarregion ausgeht und vor dem Malleolus medialis vorbeigeht.

4, Lymphstämme, die von den unteren und hinteren Teilen der Haut der Ferse ausgehen.

5, Lymphgefäße der Innenseite des Beins.

6, Lymphgefäße, von der hinteren Seite.

7, Stämme, die von außen auf die Innenseite ziehen.

8 , Stämme, die um den vorderen Teil des Knies herumgehen; sie sind bemerkenswert für ihr Volumen und besonders für ihre zahlreichen Beugungen, die im Zustand der Beugung des Gelenks verschwinden.

9, Lymphstämme des anteromedialen Teils des Oberschenkels. 10, Stämme aus dem posteromedialen Teil des Oberschenkels. 11, Stämme, die von seinem posterolateralen Teil kommen. 12, Stämme, die von den Hinterbacken stammen.

13, Stämme, die vom inneren Teil des Gesäßes und der Analregion ausgehen.

14, Stämme, die aus der Haut der Gleitbeutel austreten.

15. Anderer Stamm, der von den erektilen Teilen des Penis ausgeht.

16, Ein großer Lymphknoten, zu dem die meisten Stämme, die an der Innenseite von Bein und Oberschenkel verlaufen, gehen.

17, Ein weiterer Lymphknoten, ebenfalls groß, bei dem die meisten Stämme vom hinteren und inneren Teil des Oberschenkels enden.

18, Große Stämme, die von den vorangehenden Ganglien ausgehen und vertikal zur Vorderseite der Oberschenkelarterie ansteigen und in einem Lymphknoten enden, der sich an der 


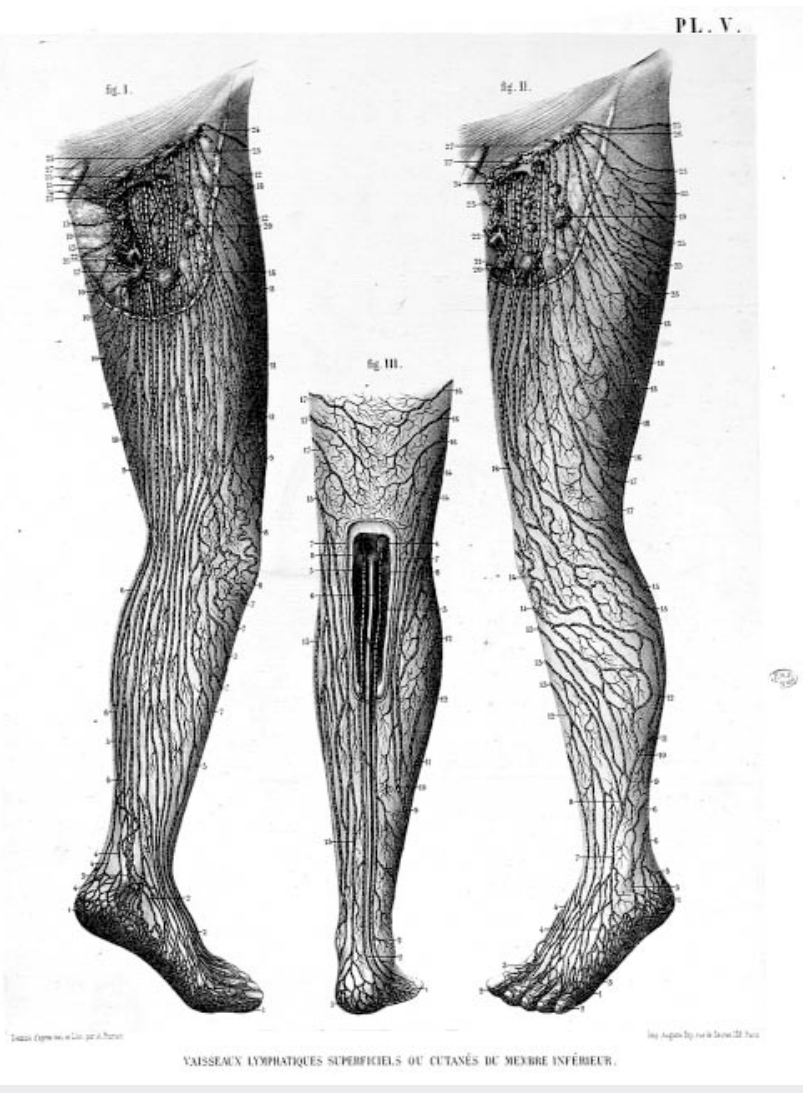

- Abb. 3 Bildtafel V aus Sappey (1874) [15].

A. iliaca externa unmittelbar oberhalb des Oberschenkelbogens befindet.

19, Ein weiterer beträchtlicher efferenter Stamm, der vor der Oberschenkelvene verläuft und in denselben Lymphknoten geht.

20, Lymphknoten, der die Gefäße des anterolateralen Teils des Oberschenkels aufnimmt.

21, Lymphknoten, in dem die meisten Gefäße des anteromedialen Teils des Oberschenkels enden.

22, Terminales Ende der V. saphena magna, von der ein Teil herausgeschnitten wurde, um die darunter liegenden Lymphstämme freizulegen.

23, Lymphknoten, zu dem die vom Penis ausgehenden Gefäße führen.

24, Lymphknoten, der fast alle Gefäße der Lendenregion und einen Teil der Gefäße der Glutealregion aufnimmt.

25, Lymphknoten, die sich auf der Verlängerung der vorherigen befinden, zu der sie durch die efferenten Ganglien, die diese verlassen, verbunden werden. Diese Lymphknoten nehmen die Gefäße der vorderen Bauchdecke auf.

26, Untere Partie der Faszie des M. obliquus externus abdominis. 27, Äußerer Leistenring, der vom Strang der Samenleiter durchzogen ist.

Abb. II - Lymphgefäße des anterolateralen Teils der unteren Extremität
1, Lymphatisches Netzwerk der Fußaußenkante; Stämme, die von diesem Netzwerk ausgehen; wenn sie sich vereinigen, ergeben sie Stämme, die schräg auf der Rückenfläche ansteigen. 2, Lymphatische Netzwerke der Peripherie der Zehen.

3, Lymphstämme, die zum Teil aus diesen Netzwerken, zum Teil aus der Metatarsalregion der Fußsohle stammen.

4, Lymphstämme der dorsalen Seite des Fußes.

5, Ursprung der Gefäße, die die V. saphena parva begleiten.

6 , Lymphstämme, die dieser Vene folgen und in den Kniekehlenganglien enden.

7, Konstant voluminöser Stamm, der über den Außenknöchel verläuft und durch seine aufeinanderfolgenden Bifurkationen fast alle Gefäße der Außenseite des Beins hervorbringt.

8 , 9, Erste und zweite Bifurkation dieses Hauptstamms. Die erste Abteilung befindet sich meist $4 \mathrm{~cm}$ oberhalb des Malleolus lateralis.

10, 11, Dritte und vierte Gabelung in der Regel sehr nahe an den vorherigen.

12, Lymphgefäße an der Außenfläche des Beins. Diese meist voluminösen Gefäße sind abwechselnd in verschiedene Richtungen gekrümmt und werden durch häufige Anastomosen verbunden.

13, Lymphstämme, die von der Außenseite zur Innenseite des Beins verlaufen und den Schienbeinkamm schräg durchschneiden.

14, Anderer Stamm, sperrig und abwechselnd in verschiedene Richtungen gekrümmt, der um den vorderen Teil des Knies herumgeht, um auch nach innen und oben getragen zu werden.

15, Zwei Lymphstämme aus der Haut des hinteren Teils des Beins.

16, Alle Stämme des anterolateralen Teils des Oberschenkels. 17, Zwei Stämme, die aus der Haut der Fossa poplitea stammen und sich nach einer kurzen Reise wieder vereinigen.

18, Stämme, die von der Rückseite des Oberschenkels ausgehen und um seine Außenseite herumgehen.

19 , Lymphknoten, in den diese vier Stämme gehen.

20, Großer Lymphknoten, der die wichtigsten Gefäße des anteromedialen Teils der Extremität aufnimmt.

21, Lymphknoten, der die Stämme des posterolateralen Teils des Oberschenkels aufnimmt.

22, Terminales Ende der langen V. saphena magna.

23, Lymphknoten, in den die Gefäße des Perineums, der Analregion und des medialen Teils des Gesäßes münden.

24, Lymphknoten, in den die meisten Lymphgefäße der äußeren Genitalorgane abfließen.

25, Lymphstamm aus der Glutealregion.

26, Lymphknoten, der diese Gefäße erhält.

27, Lymphknoten, in die die Gefäße aus dem subumbilikalen Teil der vorderen Bauchdecke einmünden.

\section{Abb. III - Lymphgefäße des hinteren Beins}

1, Lymphatisches Netz des seitlichen Fußrandes; daraus ergeben sich mehrere Stämme; die beiden wichtigsten verlaufen nach hinten: dies sind die peronealen Saphena-Stämme.

2, Diese beiden Lymphstämme befinden sich auf jeder Seite der V. saphena parva und parallel zu ihr. 
3, Stamm der äußeren peronealen Saphena-Gruppe.

4, Lymphknoten, in dem es endet.

5, Langer peronealer Saphena-Stamm, der in die Fossa poplitea eintaucht, um einen weniger oberflächlichen Lymphknoten als den vorhergehenden zu erreichen.

6. V. saphena parva.

7, Schnittrand der Haut.

8, Schnittrand der Beinfaszie, unter dem die peronealen Saphena-Stämme in der oberen Hälfte ihres Verlaufs hindurchziehen.

9, Großer Stamm, der über den Außenknöchel verläuft und durch seine aufeinanderfolgenden Bifurkationen fast alle Gefäße der Außenfläche des Beins hervorbringt.

10 , Seine zweite Bifurkation.

11, Dritte Bifurkation.

12, Alle Lymphgefäße, die aus diesem Stamm kommen.

13, Posteromediale Gefäße des Beins.

14, Zwei Stämme, die vom hinteren Teil der Extremität zum anterolateralen Teil des Oberschenkels verlaufen.

15, Ein weiterer Stamm, der, nachdem er auf dem hinteren Teil des Beins verlaufen ist, um das Knie herum bis zum medialen Teil des Oberschenkels geht.

16, Drei Stämme, die vom posteromedialen Teil des Oberschenkels ausgehen, von dem aus sie um seine Außenseite herumgehen, um seine Vorderseite zu erreichen.

17, Andere Stämme, die den gleichen Ausgangspunkt wie die vorherigen haben, die aber nicht nach außen, sondern nach innen gehen, um sich mit den Gefäßen an der Innenseite des Oberschenkels zu verbinden.

\section{Rezente Beschreibungen}

Erst in neuerer Zeit haben sich mehrere Autor*innen mit der Lagebeziehung zwischen den oberflächlichen Lymphgefäßen und der V. saphena magna beschäftigt. Zu den ersten gehörten 1995 Stefan Kubik und Mirjana Manestar [16]. Nach ihren Befunden überqueren die Kollektoren am Fußrücken die Venen, in allen übrigen Bereichen aber unterqueren sie diese. Nur die V. saphena magna wird wiederum von Kollektoren überkreuzt. Diese hat ebenfalls begleitende Lymphgefäße, ihre Adventitia selbst enthält jedoch keine Lymphgefäße. Die Position der Kollektoren hängt letztlich von der Dicke des subkutanen Fettgewebes ab. Am Fußrücken sind die Lymphgefäße oft eng mit der Lederhaut verbunden, am Unterschenkel hingegen sind sie in das Fettgewebe eingebettet. Einige Kollektoren sind eng mit den Perforansvenen verknüpft. In der Knieregion steigt das Lymphgefäßbündel dorsomedial zum medialen Kondylus des Femurs auf. Am Oberschenkel, wo das subkutane Fettgewebe dick ist, bilden die Kollektoren drei Schichten. Die oberflächlichen Leistenlymphknoten, die das Bein und die äußeren Genitalien drainieren, liegen um den Hiatus saphenus herum und sind eng mit dem subinguinalen Venenstern verbunden.

Vivien Schacht und Mitarbeiter haben 2009 erneut die Anatomie des subkutanen Lymphgefäßnetzes des menschlichen Beins in Bezug auf die V. saphena magna untersucht [4]. Es zeigte sich, dass die Lymphkollektoren am Fußrücken eng parallel zur Leder- haut verlaufen, während sich in der Leiste eine größere Zahl von Kollektoren um die V. saphena magna gruppiert. Das ventromediale Bündel, das in die oberflächlichen Inguinalknoten abfließt, umfasste 5-20 Kollektoren. Die durchschnittliche Breite des ventromedialen Bündels variierte zwischen $116 \mathrm{~mm}$ in der Mitte des Unterschenkels und $32 \mathrm{~mm}$ in der Leiste. Am Talokalkaneal-Gelenk, am Unterschenkel und am Knie waren viele Lymphkollektoren weit um die V. saphena magna verteilt, während in der Mitte des Oberschenkels und der Leiste die Lymphkollektoren innerhalb von 3-5 cm ventrolateral der V. saphena magna konzentriert waren. Schacht et al. konnten die frühere Annahme eines Engpasses des ventromedialen Bündels am Knie, des sogenannten Flaschenhalses, nicht bestätigen. Zudem konnte die Hypothese eines einzigen Wächterlymphknotens für eine bestimmte Region des Beins durch ihre Daten nicht unterstützt werden.

Das Team um Saam Tourani untersuchte 2013 die Anatomie der oberflächlichen Lymphgefäße der Bauchdecke und des Oberschenkels und ihre Auswirkungen auf die lymphatische Mikrochirurgie [3]. Die Ober- und Unterbauchkollektoren lagen oberflächlich der Fascia investiens abdominis, unmittelbar unterhalb der subdermalen Venulen. Sie waren dünnwandig und durchscheinend, und ihr Durchmesser lag zwischen 0,2 und 0,8 mm. Am Oberschenkel wurden zwei verschiedene Gruppen von oberflächlichen Kollektoren gefunden. Die Kollektoren des ventromedialen Bündels stellten die Mehrheit der oberflächlichen Kollektoren dar, waren tief im subkutanen Fettgewebe, hatten einen Durchmesser von 0,6-1,0 mm, waren dickwandig und drainierten durchgehend in zwei große Knoten inferolateral zur Crosse. Die lokalen Kollektoren des Oberschenkels waren unmittelbar unterhalb der subdermalen Venulen, 0,3-0,5 mm tief, dünnwandig und drainierten in die superolaterale Gruppe der oberflächlichen Inguinalknoten, die auch den Unterbauch, den unteren Rücken und die obere Glutealregion drainierten.

Pan Wei-Ren und Mitarbeiter untersuchten 2013 die oberflächliche Lymphdrainage der unteren Extremität [6]. Dabei beschrieben sie, dass sie zahlreiche Lymphkollektoren im subkutanen Gewebe und im oberflächlichen femoralen Gefäßbündel der unteren Extremität identifizieren konnten. Diese Kollektoren entsprangen unter der Dermis auf jeder Seite der Zehen, des Fußes und der lateralen Seite des Oberschenkels. Die Durchmesser der Gefäße variierten von 0,2 bis 2,2 mm. Die Gefäße verliefen im subkutanen Gewebe der unteren Extremität in Richtung der Kniekehlen-, Oberschenkel- sowie der oberflächlichen und tiefen Leistenlymphknoten. Während ihres gewundenen Verlaufs verzweigten, divergierten und konvergierten einige Gefäße; manchmal anastomosierten sie mit benachbarten Gefäßen oder kreuzten diese. Die meisten Gefäße konvergierten zu größeren Kollektoren und verzweigten sich dann in kleine Äste, bevor sie in die Lymphknoten eintraten.

Ein Jahr später beschrieben Pan und Mitarbeiter die unterschiedlichen Lymphabflusswege von der Ferse zur Leistenregion [17]. Dabei wurden zwei Gruppen von Lymphkollektoren identifiziert. Die mediale Gruppe, die aus der Haut zwischen dem medialen Malleolus und der Achillessehne entspringt, verlief entlang der medialen Seite des Beins und des Oberschenkels bis zu den Leistenlymphknoten. Die laterale Gruppe, die aus der Haut zwischen dem lateralen Malleolus und der Achillessehne entspringt, verlief 


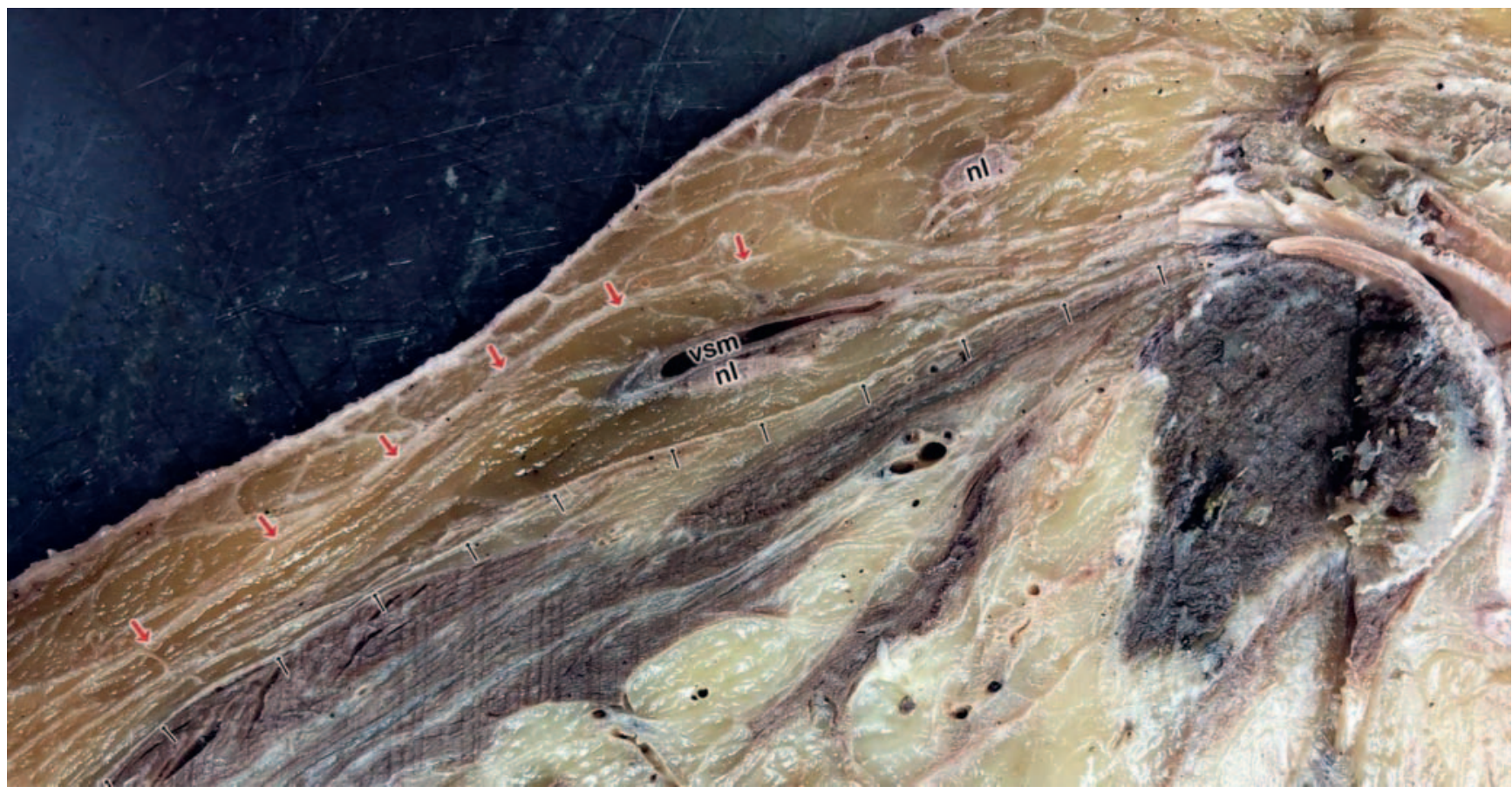

- Abb. 4 Längsschnitt durch einen Oberschenkel, annähernd parallel zur V. saphena magna. vsm: V. saphena magna; nl: Nodus lymphaticus; schwarze Pfeile: Fascia lata; rote Pfeile: Fascia saphena.

Wie bereits von Caggiati und Ricci [8] beschrieben, hebt sich die Fascia saphena von distal kommend nach proximal ab. Die ursprünglich sehr kompakte Fascia saphena löst sich in immer feinere Lamellen auf, die in ihrer Gesamtheit dann die Fascia cribrosa bilden. Eine direkte Verbindung der Fascia saphena mit dem Lig. inguinale ist nicht nachweisbar.

entlang der posterolateralen Seite des Beins bis zur Kniekehle. Dann wurden alternative Wege von der Kniekehle zu den Leistenlymphknoten identifiziert. Zahl, Größe, Art und Verteilung der Lymphgefäße und -knoten waren von Person zu Person unterschiedlich.

\section{Material und Methode}

In der hier präsentierten explorativen anatomischen Untersuchung wurden 20 untere Extremitäten von 10 Individuen beiderlei Geschlechts verwendet, die dem Institut für Klinisch-Funktionelle Anatomie der Medizinischen Universität Innsbruck zu Lebzeiten ihre Einwilligung zur Verwendung ihrer Körper für wissenschaftliche und pädagogische Zwecke erteilt hatten [18, 19].

Alle Körper wurden durch eine arterielle Injektion einer Formaldehyd-Phenol-Lösung bzw. einer Alkohol-Glycerin-Lösung und eine bis drei Monate lange Lagerung in $5 \%$ Phenol in Wasser konserviert [20].

Was die Repräsentativität betrifft, so ergab eine aktuelle Analyse, dass die dem Institut für Klinisch-Funktionelle Anatomie der Medizinischen Universität Innsbruck gespendeten Körper eine repräsentative Stichprobe der allgemeinen österreichischen Population zum Todeszeitpunkt darstellen [21].

An den rechten Präparaten wurde eine akribische und soweit wie möglich schichtweise Präparation der Subinguinalregion durchgeführt. Die linken Präparate wurden gefroren und zur Hälfte longitudinal und zur anderen Hälfte transversal geschnitten. Die dadurch erhaltenen Schnitte wurden auf den genauen
Verlauf der Fascia lata, der Fascia cribrosa und der Fascia saphena untersucht und die genaue Lage der Lymphknoten und -bahnen wurde festgestellt.

\section{Ergebnisse}

Die V. saphena magna steigt am medioventralen Oberschenkel im sogenannten Saphena-Kompartiment, gebildet von der Fascia saphena und der Fascia lata, auf. Sie wird dabei von einigen Kollektoren des ventromedialen Bündels begleitet [5]. Weitere Kollektoren dieses Bündels liegen zudem oberflächlich der Fascia saphena. Am proximalen Oberschenkel erweitert sich das Saphena-Kompartiment, die Fascia saphena hebt sich ab und wird Teil der Fascia cribrosa ( $\bullet$ Abb. 4$)$.

In diesem erweiterten Raum finden sich bis zu 20 Lymphkollektoren [4], zumeist lateral der $\mathrm{V}$. saphena magna gelegen; sie liegen sowohl oberflächlich als auch tief zur Vene. Knapp vor der Crosse, in einem Abstand von bis zu $5 \mathrm{~cm}$, finden sich mehrere Lymphknoten in engem Kontakt zur V. saphena magna, ebenfalls meist lateral ( $\triangleright$ Abb. 5); ihre Zahl schwankt individuell stark, und sie sind durch zahlreiche Lymphgefäße querverbunden, nicht aber mit jenen oberflächlichen Lymphknoten, die in die Abstrombahn der oberflächlichen Bauchwand eingebaut sind.

Die eigentlichen efferenten Lymphkollektoren dieser oberflächlichen inguinalen Lymphknoten des Beins treten zumeist mit der V. saphena magna durch den Hiatus saphenus, um dort in die tiefen inguinalen Lymphknoten zu münden ( $\bullet \mathbf{A b b}$. 6). 


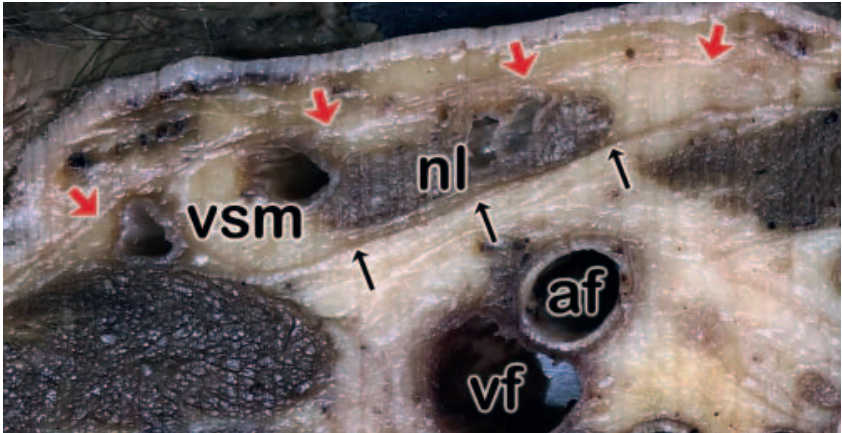

- Abb. 5 Transversalschnitt durch einen Oberschenkel ca. $5 \mathrm{~cm}$ distal der Crosse.

vsm: V. saphena magna; nl: Nodus lymphaticus; af: A. femoralis; vf: $\checkmark$ femoralis; schwarze Pfeile: Fascia lata; rote Pfeile: Fascia saphena. Die $V$ saphena magna liegt im von der Fascia lata und der Fascia saphena gebildeten Saphena-Kompartiment. Lateral der V. saphena magna, innerhalb des Saphena-Kompartiments findet sich ein großer Lymphknoten, bei dem auch aus dem Lymphknotenmark stammende Vasa lymphatica efferentia zu sehen sind. Medial der V. saphena magna - außerhalb des Saphena-Kompartiments - verläuft die V. saphena accessoria posterior.

Am Unterschenkel begleitet zudem der N. saphenus die V. saphena magna in ihrem Saphena-Kompartiment. Der N. saphenus ist ein sensibler Hautast des N. femoralis. Am Oberschenkel tritt er gemeinsam mit der A. und V. femoralis in den Adduktorenkanal ein, durchbricht aber dann die Membrana vastoadductoria und tritt von innen in den Muskelfaszientunnel des M. sartorius ein. Erst auf Höhe des Kniegelenksspalts, dort wo der M. sartorius nach ventral zum Pes anserinus superficialis umbiegt, durchbricht der N. saphenus, meist dorsal der V. saphena magna, die Fascia lata, um dann innerhalb des Saphena-Kompartiments nach distal zu verlaufen. Seine Hautäste, der R. infrapatellaris und die Rr. cutanei cruris mediales, durchbrechen in weiterer Folge die Fascia saphena und innervieren die Haut distal der Patella sowie an der medialen Seite des Unterschenkels.

\section{Diskussion}

Die oberflächliche Drainage der Lymphe des Beins erfolgt größtenteils über Lymphgefäße bzw. Lymphkollektoren, die entlang der V. saphena magna verlaufen; nur ein kleiner Teil aus der lateralen Ferse, dem Außenknöchelbereich und einem schmalen Streifen des dorsolateralen Unterschenkels mündet bereits in der Kniekehle in die tiefen Kollektoren.

Diese Lymphgefäße müssen selbstverständlich die anfallende Lymphlast bewältigen; in der Regel - wie überall - steht eine große Reservekapazität zur Verfügung [22-24]; es gibt keine detaillierten Angaben, aber Schätzungen gehen davon aus, dass das normale physiologische Ruhelymphzeitvolumen nur etwa $20 \%$ der maximalen Transportkapazität ausmacht, manche Autoren geben sogar nur $10 \%$ an [25]. Diese große Reservekapazität ist notwendig, um momentane und kurzfristige Erhöhungen der Lymphlast, etwa durch Entzündungen oder Traumata, aufzufangen und abtransportieren zu können. Fallen einzelne Lymphgefäße aus, so können die verbliebenen Lymphgefäße deren Trans-

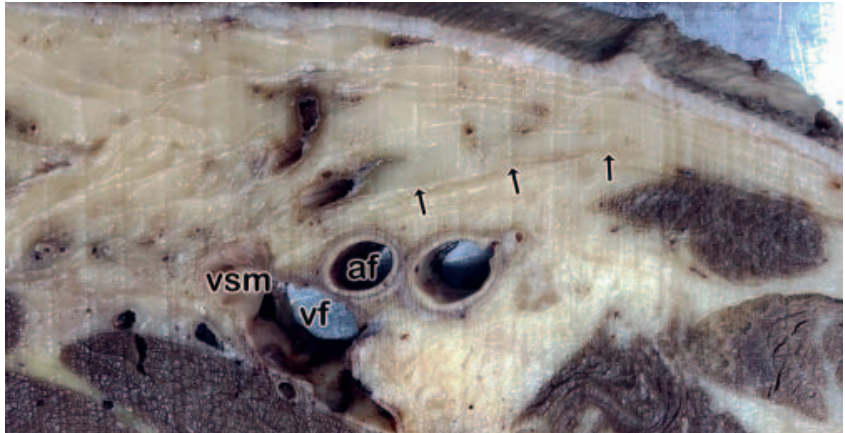

- Abb. 6 Transversalschnitt durch einen Oberschenkel auf Höhe der Crosse.

vsm: V. saphena magna; af: A. femoralis; vf: V. femoralis; schwarze Pfeile: Fascia lata.

Der Schnitt liegt unmittelbar auf Höhe der Mündung der V. saphena magna in die V. femoralis, die hier von zahlreichen kleinlumigen Lymphgefäßen umgeben ist. Eine eindeutige Fascia saphena ist nicht vorhanden; die einzelnen subkutanen Bindegewebslamellen bilden die Fascia cribrosa.

portfunktion übernehmen; dann allerdings steigt die jeweilige Transportleistung der verbliebenen Lymphgefäße. Ist diese Erhöhung der Transportleistung nur gering oder temporär, werden keine weiteren Folgen auftreten; ist die Erhöhung jedoch größer und langfristiger - eventuell sogar dauerhaft -, kommt es zu einer „Erschöpfung“ der mehr arbeitenden Lymphgefäße, die Transportkapazität sinkt, und wenn diese unter die anfallende Lymphlast sinkt, resultiert ein Lymphödem. Es ist in den seltensten Fällen anzunehmen, dass der Eingriff an der V. saphena magna allein für das Entstehen eines Lymphödems verantwortlich ist. Zum einen kann eine - bislang unerkannte - Vorschädigung des Lymphgefäßsystems bestehen, die die maximale Transportkapazität bereits reduziert hat, ohne dass daraus bereits ein Lymphödem resultiert. Zum anderen kann es nach dem Eingriff an der V. saphena magna und unabhängig davon zu einer weiteren Reduktion der maximalen Transportkapazität kommen. Die Wahrscheinlichkeit für ein Lymphödem ergibt sich demnach aus (1) dem Grad einer möglichen Vorschädigung des Lymphgefäßsystems, (2) dem Grad der Schädigung der Lymphgefäße durch den Eingriff an der V. saphena magna und (3) dem Grad einer späteren Nachschädigung des Lymphgefäßsystems.

Für die in der Einleitung genannten 2-Monats-Daten kann eine vom Eingriff unabhängige Nachschädigung weitestgehend ausgeschlossen werden. Es war also der Eingriff am Venensystem, der Lymphgefäße geschädigt, die maximale Transportkapazität unter die für die wohl schon erhöhte Lymphlast notwendige Transportkapazität gedrückt und somit das Lymphödem ausgelöst hat.

Es soll hier nochmals hervorgehoben werden, dass es im Verlauf des Lymphabflusses des Beins keinen sogenannten Flaschenhals gibt, weder am Knie [4] noch an einer sonstigen Stelle.

Einige der bisherigen Studien haben die Relation dieser Lymphgefäße zur V. saphena magna untersucht $[4,16]$, aber keine Studie hat bislang die Relation dieser Lymphgefäße zur Fascia saphena beleuchtet. Nach den Untersuchungen des Autors verlaufen die Lymphkollektoren sowohl unter- als auch oberhalb der Fascia saphena. Es kann also festgehalten werden, dass die Lymphgefäße 
zumeist lateral der V. saphena magna - sowohl oberflächlich als auch tief zur Vene - liegen.

Eine extensive Intervention an der proximalen V. saphena magna, sei es bei der klassischen Crossektomie oder bei diversen endovenösen Verfahren, deren jeweiliges Agens über die Venenwand selbst hinausreichen kann, kann zu einer zumindest teilweisen Schädigung von begleitenden Lymphgefäßen führen. Zumeist wird wohl die Gesamttransportkapazität der oberflächlichen Lymphbahnen des Beins nicht unter die anfallende Lymphlast gesenkt; jedoch kann eine vorbestehende Beeinträchtigung oder auch eine später auftretende weitere Minderung der maximalen Transportkapazität in einem Lymphödem resultieren.

Aus anatomischer Sicht wäre also ein medialer Zugang beim chirurgischen Zugang zur proximalen V. saphena magna und der Crosse selbst empfehlenswert. Darüber hinaus ist es vermutlich möglich, das subinguinale Lymphknotenpaket mit seinen einmündenden Lymphgefäßen und seinen Quervernetzungen zu umgehen, indem man ausgehend von der Hautinzision recht oberflächlich stumpf nach distal-medial präpariert, um dann in einigem Abstand von der Crosse in die Tiefe auf die eigentliche Fascia lata zu gehen. Dann kann man auf der Fascia lata wieder nach proximal in Richtung Crosse präparieren und so die lateral und oberflächlich liegenden Lymphgefäße schonen [26].

Endoluminale thermische Verfahren hingegen bergen das Risiko, jene Lymphbahnen mit zu schädigen, die in unmittelbarer Nähe der V. saphena magna liegen. Eine „anatomische Alternative“ ist nicht möglich, da diese Verfahren ja direkt in der Vene wirksam sind. Eine Schonung der anliegenden Lymphgefäße ist nur möglich, wenn die aufgewendete Energie möglichst gering gehalten wird; dies geht aber sicherlich zulasten der Verschlussrate. Der Einsatz einer Tumeszenz mag einzelne Lymphgefäße von der V. saphena magna abdrängen; dafür gibt es aber keinerlei Daten oder Studien.

\section{Interessenkonflikte}

Diese Veröffentlichung entstand auf der Basis eines eingeladenen Vortrags auf der 61. Jahrestagung der Deutschen Gesellschaft für Phlebologie 2019 in Münster (D) [31]. Für diesen Vortrag erhielt der Autor einen Reisekostenzuschuss.

Literatur

[1] Pittaluga P, Chastanet S. Lymphatic complications after varicose veins surgery: risk factors and how to avoid them. Phlebology 2012; 27 (Suppl. 1): 139-142. doi:10.1258/phleb.2012.012S12

[2] Flessenkamper I, Hartmann M, Stenger D et al. Endovenous laser ablation with and without high ligation compared with high ligation and stripping in the treatment of great saphenous varicose veins: initial results of a multicentre randomized controlled trial. Phlebology 2013; 28: 16-23. doi:10.1258/phleb.2011.011147

[3] Tourani SS, Taylor GI, Ashton MW. Anatomy of the superficial lymphatics of the abdominal wall and the upper thigh and its implications in lymphatic microsurgery. J Plast Reconstr Aesthet Surg 2013; 66: 1390-1395. doi:10.1016/j.bjps.2013.05.030

[4] Schacht V, Luedemann W, Abels C et al. Anatomy of the subcutaneous lymph vascular network of the human leg in relation to the great saphenous vein. Anat Rec (Hoboken) 2009; 292: 87-93. doi:10.1002/ar.20765
[5] Tourani SS, Taylor GI, Ashton MW. Understanding the three-dimensional anatomy of the superficial lymphatics of the limbs. Plast Reconstr Surg 2014; 134: 1065-1074. doi:10.1097/PRS.0000000000000640

[6] Pan WR, Wang DG, Levy SM et al. Superficial lymphatic drainage of the lower extremity: anatomical study and clinical implications. Plast Reconstr Surg 2013; 132: 696-707. doi:10.1097/PRS.0b013e31829ad12e

[7] Federative Committee on Anatomical Terminology (FCAT), Hrsg. Terminologia Anatomica. Stuttgart, New York: Thieme; 1998

[8] Caggiati A, Ricci S. The Long Saphenous Vein Compartment. Phlebology: The Journal of Venous Disease 2016; 12: 107-111. doi:10.1177| 026835559701200307

[9] Caggiati A. The nomenclature of the veins of the lower limbs, based on their planar anatomy and fascial relationships. Acta Chir Belg 2004; 104: 272-275. doi:10.1080/00015458.2004.11679553

[10] Cruikshank W. The Anatomy of the Absorbing Vessels of the Human Body. London: G. Nicol; 1786

[11] Mascagni P. Vasorum Lymphaticorum Corporis Humani Historia et Ichnographia. Senis (Siena): Pazzini Carli; 1787

[12] Schnalke T. Der expandierte Mensch - Zur Konstitution von Körperbildern in anatomischen Sammlungen des 18. Jahrhunderts. In: Stahnisch F, Steger F, Hrsg. Medizin, Geschichte und Geschlecht Körperhistorische Rekonstruktionen von Identitäten und Differenzen. Stuttgart: Franz Steiner Verlag; 2005: 63-82

[13] Ludwig CF. William Cruikshank's Geschichte und Beschreibung der einsaugenden Gefäße oder Saugadern des menschlichen Körpers. Leipzig: Weidmann; 1789

[14] Ludwig CF. Paul Mascagni's Geschichte und Beschreibung der einsaugenden Gefäße oder Saugadern des menschlichen Körpers. Leipzig: Weidmann; 1789

[15] Sappey PC. Anatomie, physiologie, pathologie des vaisseaux lymphatiques considérés chez l'homme et les vertébrés. Paris: A Delahaye; 1874

[16] Kubik S, Manestar M. Topographic relationship of the ventromedial lymphatic bundle and the superficial inguinal nodes to the subcutaneous veins. Clin Anat 1995; 8: 25-28. doi:10.1002/ca.980080104

[17] Pan WR, Levy SM, Wang DG. Divergent lymphatic drainage routes from the heel to the inguinal region: anatomic study and clinical implications. Lymphat Res Biol 2014; 12: 169-174. doi:10.1089/Irb.2014.0004

[18] McHanwell S, Brenner E, Chirculescu ARM et al. The legal and ethical framework governing Body Donation in Europe - A review of current practice and recommendations for good practice. Eur J Anat 2008; 12 : $1-24$

[19] Riederer BM, Bolt S, Brenner E et al. The legal and ethical framework governing Body Donation in Europe - 1st update on current practice. Eur J Anat 2012; 16: 1-21

[20] Platzer W, Putz R, Poisel S. Ein neues Konservierungs- und Aufbewahrungssystem für anatomisches Material. Acta Anat (Basel) 1978; 102: 60-67

[21] Konschake M, Brenner E. "Mors auxilium vitae"-Causes of death of body donors in an Austrian anatomical department. Ann Anat 2014; 196: 387-393. doi:10.1016/j.aanat.2014.07.002

[22] Al-Kofahi M, Yun JW, Minagar A et al. Anatomy and roles of lymphatics in inflammatory diseases. Clinical and Experimental Neuroimmunology 2017; 8: 199-214. doi:10.1111/cen3.12400

[23] Foldi M. Lymph Formation and Lymph Flow: "Physiological Lymph Drainage". In: Földi M, Strößenreuther R, Hrsg. Foundations of Manual Lymph Drainage. Saint Louis: Mosby; 2005: 28-37. doi:10.1016/b978032303064-9.50008-6

[24] Wiig H, Swartz MA. Interstitial fluid and lymph formation and transport: physiological regulation and roles in inflammation and cancer. Physiol Rev 2012; 92: 1005-1060. doi:10.1152/physrev.00037.2011

[25] Földi E, Baumeister RGH, Bräutigam P et al. Zur Diagnostik und Therapie des Lymphödems. Dtsch Ärztebl 1998; 95: A-740-747 
[26] Meyer-Berendes B. UMIC - Ultraschall assistierte minimal invasive Crossektomie. 60 Jahrestagung der Deutschen Gesellschaft für Phlebologie. September 26-29, 2018.2018; Bielefeld (D)

[27] Cavezzi A, Frullini A, Ricci S et al. Treatment of varicose veins by foam sclerotherapy: Two clinical series. Phlebology 2002; 17: 13-18. doi:10.1007/bf02667958

[28] De Maeseneer MG, Philipsen TE, Vandenbroeck CP et al. Closure of the cribriform fascia: an efficient anatomical barrier against postoperative neovascularisation at the saphenofemoral junction? A prospective study. Eur J Vasc Endovasc Surg 2007; 34: 361-366. doi:10.1016/j. ejvs.2007.03.020

[29] De Maeseneer MG, Vandenbroeck CP, Lauwers PR et al. Early and late complications of silicone patch saphenoplasty at the saphenofemoral junction. J Vasc Surg 2006; 44: 1285-1290. doi:10.1016/j. jvs.2006.08.012
[30] Figueiredo M, Araujo S, Barros NJr et al. Results of surgical treatment compared with ultrasound-guided foam sclerotherapy in patients with varicose veins: a prospective randomised study. Eur J Vasc Endovasc Surg 2009; 38: 758-763. doi:10.1016/j.ejvs.2009.07.015

[31] Brenner E. Lymphbahnen an den Beinen: Hier muss die Phlebologin/der Phlebologe aufpassen. Vortrag, geladen: 61 Jahrestagung der Deutschen Gesellschaft für Phlebologie. September 18-2 1, 2019 2019; Münster (D) 